\title{
ANALYSIS OF A NUMERICAL SOLVER FOR RADIATIVE TRANSPORT EQUATION
}

\author{
HAO GAO AND HONGKAI ZHAO
}

\begin{abstract}
We analyze a numerical algorithm for solving radiative transport equation with vacuum or reflection boundary condition that was proposed by the authors in 2009 with angular discretization by the finite element method and spatial discretization by the discontinuous Galerkin method or the finite difference method.
\end{abstract}

\section{INTRODUCTION}

Radiative transport equation (RTE) (1.1) models a wide range of applications, such as neutron transport, atmospheric radiative transfer, heat transfer and photon migration in tissues. In this paper, we study a numerical algorithm for the following RTE on a bounded domain $\Omega$ with smooth boundary $\partial \Omega$,

$$
\hat{s} \cdot \nabla \Phi(\vec{x}, \hat{s})+\mu_{t} \Phi(\vec{x}, \hat{s})=\mu_{s} \oint f\left(\hat{s}, \hat{s}^{\prime}\right) \Phi\left(\vec{x}, \hat{s}^{\prime}\right) d \hat{s}^{\prime}+q(\vec{x}, \hat{s}), \quad(\vec{x}, \hat{s}) \in \Omega \times S,
$$

where $\Phi(\vec{x}, \hat{s})$ is the particle density at position $\vec{x}$ along direction $\hat{s}, q$ is the source term, $S$ denotes the angular space, i.e., unit circle in two dimensions (2D) $S^{1}$ or unit sphere in three dimensions (3D) $S^{2}$.

The parameters in (1.1) are absorption coefficient $\mu_{a}$, scattering coefficient $\mu_{s}$, transport coefficient $\mu_{t}=\mu_{a}+\mu_{s}$ and scattering kernel $f$. We assume all the parameters in (1.1) are non-negative and bounded. In particular, $\mu_{a}$ is bounded below by a positive constant $C_{\mu}$, i.e., $0<C_{\mu} \leq \mu_{a}$.

Very often $f$ is rotationally invariant, i.e., $f\left(\hat{s}, \hat{s}^{\prime}\right)=f\left(\hat{s} \cdot \hat{s}^{\prime}\right)$, and is normalized with $\oint f\left(\hat{s} \cdot \hat{s}^{\prime}\right) d \hat{s}^{\prime}=1$. Although we assume this rotational invariance in this paper for simplicity, our algorithm and analysis can be extended for the general case. As an example, the commonly used $f$ in optical imaging to model photon transport in tissues is the following Henyey-Greenstein (H-G) function

$$
f\left(\hat{s} \cdot \hat{s}^{\prime}\right)= \begin{cases}\frac{1-g^{2}}{2 \pi\left(1+g^{2}-2 g \hat{s}^{2} \cdot \hat{s}^{\prime}\right)}, & n=2, \\ \frac{1-g^{2}}{4 \pi\left(1+g^{2}-2 g \hat{s} \cdot \hat{s}^{\prime}\right)^{3 / 2}}, & n=3,\end{cases}
$$

where the parameter $0 \leq g \leq 1$ indicates how forward peaking the scattering is.

To specify the boundary condition, we define $\hat{n}(\vec{x})$ as the boundary normal for $\vec{x} \in \partial \Omega, \Gamma^{+}\left(\Gamma^{-}\right)=\{(\vec{x}, \hat{s}) \in \partial \Omega \times S$, with $\hat{s} \cdot \hat{n}>0(\leq 0)\}$ as the outgoing (incoming)

Received by the editor September 13, 2011.

2010 Mathematics Subject Classification. Primary 65N12.

Key words and phrases. Radiative transfer equation, discrete ordinate method, source iteration, Henyey-Greenstein function, discontinuous Galerkin method.

The work was partially supported by the NIH/NIBIB grant EB013387 and NSF grant DMS0811254. 
boundary, and $n_{i}\left(n_{o}\right)$ as the refraction index of the medium (environment). Then the reflection boundary condition for (1.1) is

$$
\Phi(\vec{x}, \hat{s})=R\left(\vec{x}, \hat{s}, \hat{s}^{\prime}\right) \Phi\left(\vec{x}, \hat{s}^{\prime}\right), \quad(\vec{x}, \hat{s}) \in \Gamma^{-},\left(\vec{x}, \hat{s}^{\prime}\right) \in \Gamma^{+},
$$

where $\hat{s}^{\prime}$ refers to angular direction at $\vec{x} \in \partial \Omega$ that is reflected into $\hat{s}$ at $\vec{x}$ when $n_{i} \neq n_{o}$, and $R$ corresponds to the reflection energy ratio computed through the Fresnel formula [5]. When there is no refraction index mismatch, i.e., $n_{i}=n_{o},(1.3)$ is the so-called vacuum boundary condition with $R=0$.

With the aforementioned conditions on $\mu_{a}, \mu_{s}$ and $f$, there exists a unique positive solution to RTE (1.1), when $Q(\vec{x}, \hat{s})=\int_{0}^{d} q(\vec{x}-r \hat{s}, \hat{s}) e^{-\int_{0}^{r} \mu_{t}\left(\vec{x}-r^{\prime} \hat{s}\right) d r^{\prime}} d r$ is bounded and positive [6]. Here $d$ is the distance from $\vec{x}$ to the domain boundary $\partial \Omega$ along the direction $-\hat{s}$.

In this work we will analyze the numerical algorithm proposed in [4 for RTE that works for general geometry and general reflection boundary condition. In this algorithm we use angular discretization by finite element method and spatial discretization by the discontinuous Galerkin method or the finite difference method. In Section 2 we give a brief description of the numerical method and then present various error estimates and convergence analysis in Section 3 . One of the main difficulties in our analysis comes from the general reflection boundary condition.

\section{Numerical ALGORITHMS}

The analytical solution for RTE is rarely available except in a very special setup. Efficient numerical algorithms are needed for many applications. However, the efficient numerical computation of RTE is challenging mainly due to the following difficulties:

- high dimensionality;

- different solution behavior, e.g., from the transport regime near the source to the diffusion regime after a few mean free path;

- forward peaking due to anisotropic scattering kernel function;

- general boundary conditions.

Moreover, a large and not so sparse (due to the scattering term) linear system has to be solved after discretization that requires an efficient matrix solver.

In [4, we developed an efficient multigrid numerical algorithm for RTE that works for general geometry and general reflection boundary condition. The solver is available at http://sites.google.com/site/rtefastsolver/. The key features of our method include:

- angular and spatial discretization that (1) preserves the correct behavior of transport and scattering operator, (2) captures both short and long range interactions in spatial and angular domain effectively when using the multigrid strategy;

- improved source iteration (ISI) based on Gauss-Seidel iterations with angledependent ordering of spatial nodes which (1) captures transport efficiently, (2) converges faster than source iteration (SI);

- multigrid method in both angular and spatial domain with ISI as the relaxation which further accelerates the solution algorithm, particularly in the diffusion regime;

- flexibility for both structured and unstructured grids in 2D and 3D with general boundary conditions. 
In the following, we will briefly describe our solution algorithms for RTE and the details can be found in 4 .

2.1. Angular discretization. Let $\left\{L_{m}(\hat{s}), 1 \leq m \leq M\right\}$ be the piecewise linear angular basis on an angular mesh $\left\{\hat{s}_{m}, 1 \leq m \leq M\right\}$ of $S$, i.e., $L_{m}\left(\hat{s}_{m^{\prime}}\right)=\delta_{m m^{\prime}}$, and $w_{m}=\oint L_{m} d \hat{s}$. Then we approximate the scattering angular integration $I=$ $\oint f\left(\hat{s} \cdot \hat{s}^{\prime}\right) \Phi\left(\hat{s}^{\prime}\right) d \hat{s}^{\prime}$ by

$$
\tilde{I}=\oint \tilde{f} \tilde{\Phi} d \hat{s}^{\prime}
$$

where $\tilde{f}$ and $\tilde{\Phi}$ are the piecewise linear approximation of $f$ and $\Phi$ respectively.

We start with angular discretization (Figure 1) for 2D spatial domain, where $S^{1}$ is equally divided into angular intervals $\left\{\theta_{m}, 1 \leq m \leq M\right\}$ with interval length $h_{a}$, and therefore is also rotationally invariant in terms of $h_{a}$. The angular discretization of the scattering summation by the finite element method (FEM) goes as follow. For each $\hat{s}_{m}, \tilde{I}=\oint \tilde{f}\left(\theta_{m^{\prime}}-\theta_{m}\right) \tilde{\Phi}\left(\theta_{m^{\prime}}\right) d \theta_{m^{\prime}}$. Moreover, since $\tilde{\Phi}=\sum_{m^{\prime}} \Phi_{m^{\prime}} L_{m^{\prime}}$, we have $\tilde{I}=\sum_{m^{\prime}} w_{m m^{\prime}}^{0} \Phi_{m^{\prime}}$ with $w_{m m^{\prime}}^{0}=\oint \tilde{f}\left(\theta_{m^{\prime}}-\theta_{m}\right) L_{m^{\prime}} d \theta_{m^{\prime}}$. Then we normalize the weight $w_{m m^{\prime}}^{0}$ 's to get $w_{m m^{\prime}}$ 's, i.e., $w_{m m^{\prime}}=w_{m m^{\prime}}^{0} /\left(\sum_{m^{\prime}} w_{m m^{\prime}}^{0}\right)$ so that $\sum_{m^{\prime}} w_{m m^{\prime}}=$ 1. In summary, the angular scattering summation is discretized as

$$
I^{h_{a}}=\sum_{m^{\prime}=1}^{M} w_{m m^{\prime}} \Phi_{m^{\prime}}, 1 \leq m \leq M .
$$

Please note that $w_{m m^{\prime}}=w_{m^{\prime} m}$ due to the rotational invariance of $f$ and the uniformity of the angular mesh.

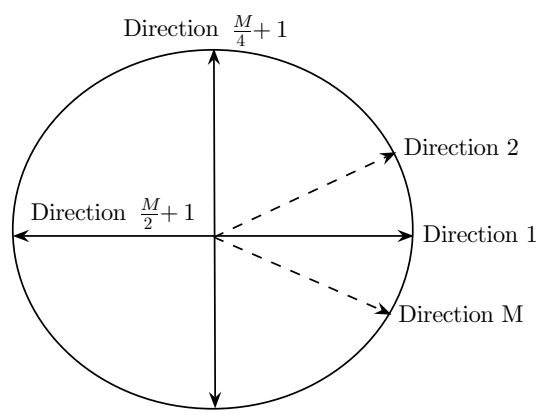

Figure 1. Angular discretization in 2D

In $3 \mathrm{D}$, the angular mesh on the unit sphere $S^{2}$ is the projection of eight triangular planes, each of which belongs to a different quadrant. The mesh on the triangular plane in the first quadrant is shown in Figure 2. And then the mesh is projected onto the unit sphere which gives a polyhedral approximation of the sphere. This simple triangulation is quite uniform although it is not rotationally invariant. Moreover, it is easy to coarsen and refine for multigrid method in angular domain. The discretization is similar to $2 \mathrm{D}$ and the weight $w_{m m^{\prime}}^{0}$ is computed on the polydedron with the piecewise linear approximation $\tilde{f}$ defined on each triangular element of the polyhedron. Then the weight $w_{m m^{\prime}}$ is normalized from $w_{m m^{\prime}}^{0}$ as in $2 \mathrm{D}$. Note that $w_{m m^{\prime}}=w_{m^{\prime} m}$ is not the case in $3 \mathrm{D}$ due to the violation of the rotation invariance of the angular mesh in 3D. 


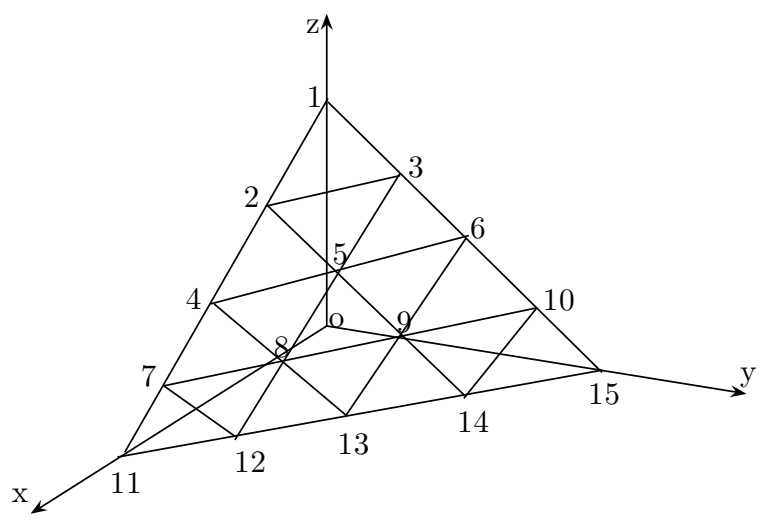

Figure 2. Angular discretization in 3D

Comparing with the angular discretization through spherical harmonic expansion, the direct use of an angular mesh allows to (1) capture both short and long range interactions effectively when using the multigrid method, (2) deal with general reflection boundary condition at ease, which consequently is transformed into some local dependence in angular domain, e.g., through Snell's law. Moreover, piecewise linear approximation guarantees that $w_{m m^{\prime}} \geq 0$, which preserves the qualitative behavior of localized scattering even if the angular mesh resolution may not be enough in an extremely forward peaking situation. We will estimate the error between $\phi$ after the angular discretization (2.3) and RTE solution $\Phi$ in section 3.1 .

Remark 2.1. Although an octahedron with eight equilateral triangular faces are used for angular discretization, for better uniformity, an icosahedron, a regular polyhedron with 20 identical equilateral triangular faces, can be used.

2.2. Spatial discretization. Let $\left\{\phi_{m}(\vec{x}), 1 \leq m \leq M\right\}$ be the RTE solution along the angular grid $\left\{\theta_{m}, 1 \leq m \leq M\right\}$ after angular discretization. (1.1) is now the following coupled system of first-order partial differential equations in space

$$
\hat{s}_{m} \cdot \nabla \phi_{m}+\mu_{t} \phi_{m}=\mu_{s} \sum_{m^{\prime}=1}^{M} w_{m m^{\prime}} \phi_{m^{\prime}}+q_{m}, 1 \leq m \leq M,
$$

where $q_{m}$ is the source term along the direction $\hat{s}_{m}$, i.e., $q_{m}=q\left(\vec{x}, \hat{s}_{m}\right)$, and the scattering integral is approximated by the summation with the scattering weight $\left\{w_{m m^{\prime}}, 1 \leq m, m^{\prime} \leq M\right\}$. Notice that $w_{m m^{\prime}}$ 's are all positive since they are computed through piecewise linear FEM for non-negative functions.

Next we discretize the system (2.3) in space. In [4, we chose the Discontinuous Galerkin (DG) method for the unstructured grid (see section 3.2 for the DG discretization). DG was initially developed for scalar transport equation [9] for neutron transport, and it deals with hyperbolic problems such as transport equation effectively due to its natural upwind nature as well as flexibility for high-order approximation and mesh geometry. Besides, in implementation there is no need to form global matrix explicitly for DG and the solution is solved by successively sweeping through individual mesh elements. We will analyze DG discretization of 
the system (2.3) on unstructured mesh in section 3.2. Recently, the DG method was also proposed for spatial discretization for RTE in 8 in which the discrete ordinate method is used for angular discretization. An error estimate is provided for the discrete ordinate DG method for the vacuum boundary condition.

On the other hand, we chose the finite difference method (FDM) for the structured grid [4]. For example, with the first-order upwind FDM on the 2D spatial domain when $\theta_{m} \in\left[0, \frac{\pi}{2}\right)$, we have

$$
\left(a+b+\mu_{t}\right) \phi_{i, j, m}-\left(a \phi_{i-1, j, m}+b \phi_{i, j-1, m}\right)-\mu_{s} \sum_{m^{\prime}=1}^{M} w_{m m^{\prime}} \phi_{i, j, m^{\prime}}=q_{i, j, m}
$$

where $a=\frac{\cos \theta_{m}}{\triangle x} \geq 0, b=\frac{\sin \theta_{m}}{\triangle y} \geq 0$, and $i, j$ are indices for $x$ and $y$ coordinates, respectively. We will analyze FDM for the system (2.3) on structured mesh in section 3.3 .

2.3. Improved source iteration (ISI). SI (source iteration) is a popular method for solving the discretized linear system of RTE. In SI, the scattering entirely lags one step behind transport, i.e.,

$$
\left(\hat{s} \cdot \nabla+\mu_{t}\right) \Phi^{n+1}(\vec{x}, \hat{s})=\mu_{s} \oint f\left(\hat{s}, \hat{s}^{\prime}\right) \Phi^{n}\left(\vec{x}, \hat{s}^{\prime}\right) d \hat{s}^{\prime}+q(\vec{x}, \hat{s}) .
$$

This simple iterative procedure has a clear physical interpretation, e.g., $\Phi^{n}$ captures all particles that have been scattered no more than $n$ times. However, SI has a slow convergence when the scattering is dominant, e.g., in the optical thick regime where the domain size is large in terms of mean free paths.

ISI was developed in 4. ISI is essentially a Gauss-Seidel method in both angular and spatial variables, which utilizes the most updated information during iterations. We also use angle-dependent ordering in ISI, i.e., the ordering that aligns with $\hat{s}$ to follow the characteristic direction of the transport operator. For simplicity, we illustrate SI and ISI using the first-order upwind FDM (2.4). Here since $\theta_{m} \in\left[0, \frac{\pi}{2}\right)$, the ordering in ISI is $i=1,2, \cdots, j=1,2, \cdots$. Then ISI is

$$
\begin{aligned}
\phi_{i, j, m}^{n}= & \frac{a \phi_{i-1, j, m}^{n}+b \phi_{i, j-1, m}^{n}}{a+b+\mu_{t}-\mu_{s} w_{m m}} \\
& \frac{+\mu_{s_{i j}}\left(\sum_{m^{\prime}=1}^{m-1} w_{m m^{\prime}} \phi_{i, j, m^{\prime}}^{n}+\sum_{m^{\prime}=m+1}^{M} w_{m m^{\prime}} \phi_{i, j, m^{\prime}}^{n-1}\right)+q_{i, j, m}}{a+b+\mu_{t}-\mu_{s} w_{m m}},
\end{aligned}
$$

while the SI is

$$
\phi_{i, j, m}^{n}=\frac{a \phi_{i-1, j, m}^{n}+b \phi_{i, j-1, m}^{n}+\mu_{s_{i j}} \sum_{m^{\prime}=1}^{M} w_{m m^{\prime}} \phi_{i, j, m^{\prime}}^{n-1}+q_{i, j, m}}{a+b+\mu_{t}} .
$$

It can be easily shown that upwind spatial discretization combined with angular discretization for general boundary condition (2.8) produces a linear system with the $M$-matrix, which guarantees that (1) ISI converges and converges faster than SI, (2) global error in maximum norm satisfies maximum principle, (3) ISI is a smoothing operation. In section 3.3, we will analyze this FDM scheme (2.4) and compare ISI (2.6) with SI (2.7). With correct ordering ISI can capture the solution in transport regime efficiently. On the other hand, multiple scattering, which causes diffusive behavior in both angular and spatial domain, can be handled by our multigrid (in both angle and space) approach effectively. 
2.4. Boundary condition. Our direct FEM-based angular discretization deals with general boundary conditions at ease. For example, if there is a mismatch of refraction index at the boundary, local mesh-based photon flux in one direction can be reflected and refracted into other directions according to Fresnel formula [5]. To discretize (1.3) at a boundary point $\vec{x}$ along an incoming direction $\hat{s}_{m}$, which belongs to the angular mesh, there is a outgoing direction $\hat{s}^{\prime}$ such that $\phi\left(\vec{x}, \hat{s}_{m}\right)=R\left(\vec{x}, \hat{s}_{m}, \hat{s}^{\prime}\right) \Phi\left(\vec{x}, \hat{s}^{\prime}\right)$, where $\left(\vec{x}, \hat{s}_{m}\right) \in \Gamma^{-},\left(\vec{x}, \hat{s}^{\prime}\right) \in \Gamma^{+}$. However, $\hat{s}^{\prime}$ may not belong to the angular mesh, so we use linear interpolation of fluxes at neighboring angular mesh points $\hat{s}_{m^{\prime}}$ to approximate the flux in direction $\hat{s}^{\prime}$. So after angular discretization, the reflection boundary condition becomes

$$
\phi_{m}(\vec{x})=\sum_{m^{\prime}} r_{m m^{\prime}}(\vec{x}) \phi_{m^{\prime}}(\vec{x}), \quad\left(\vec{x}, \hat{s}_{m}\right) \in \Gamma^{-},\left(\vec{x}, \hat{s}_{m^{\prime}}\right) \in \Gamma^{+} .
$$

Two neighboring directions whose interval encloses $\hat{s}^{\prime}$ in 2D and three neighboring directions whose triangle encloses $\hat{s}^{\prime}$ in $3 \mathrm{D}$ are used for the linear interpolation. In particular, $\sum_{m^{\prime}} r_{m m^{\prime}}=R\left(\vec{x}, \hat{s}_{m}, \hat{s}^{\prime}\right) \leq 1$.

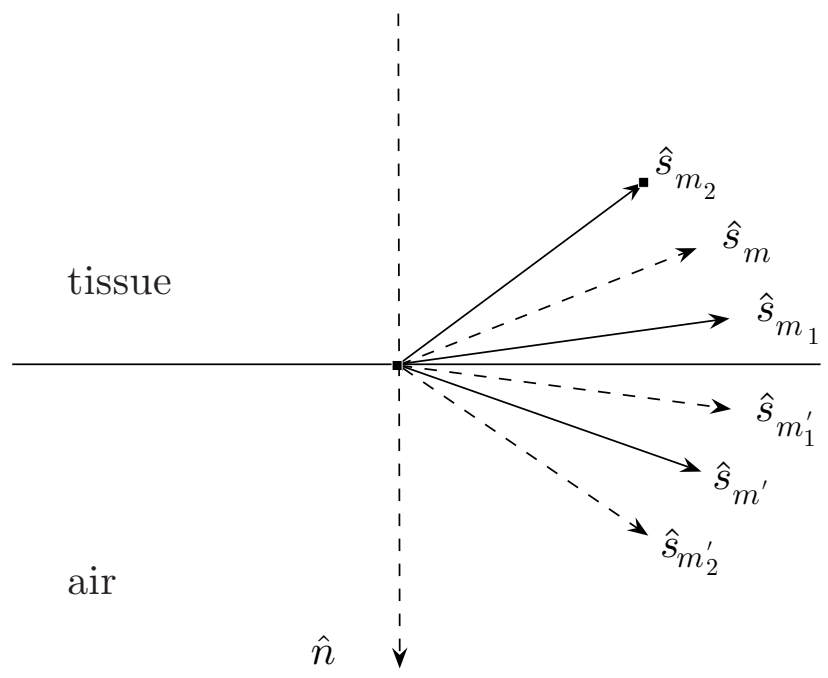

FiguRE 3. Reflection at the boundary in 2D: only the solid lines are on the angular grid.

When the incoming direction $\hat{s}_{m}$ is almost tangential to the boundary at $\vec{x}$, the corresponding outgoing direction $\hat{s}^{\prime}$ is also almost tangential to the boundary. Thus some $\hat{s}_{m^{\prime}}$ used in the interpolation may be an incoming direction. See Figure 3 for a $2 \mathrm{D}$ example: $\hat{s}_{m_{1}^{\prime}}$ is the reflection angle for $\hat{s}_{m_{1}}$, which is not on the angular grid and is interpolated from $\hat{s}_{m_{1}}$ and $\hat{s}_{m^{\prime}}$. In $2 \mathrm{D}$ it is easy to show that the general form (2.8) is still valid. That is $\phi_{m}(\vec{x})=r\left[\alpha \phi_{m}(\vec{x})+(1-\alpha) \phi_{m^{\prime}}(\vec{x})\right]$, where $r=R\left(\vec{x}, \hat{s}_{m}, \hat{s}^{\prime}\right) \leq 1$ and the weight $\alpha>0$ in the linear interpolation. So

$$
\phi_{m}(\vec{x})=\frac{r(1-\alpha)}{1-r \alpha} \phi_{m^{\prime}}(\vec{x}) \leq r \phi_{m^{\prime}}(\vec{x}) .
$$

In $3 \mathrm{D}$ the situation is more complicated since three neighboring directions are involved in the linear interpolation. Incoming directions that are almost tangential to 
the boundary may not be explicitly represented in terms of outgoing directions, e.g., when two of the three directions used in the interpolation are incoming directions.

In summary, the general reflection boundary condition (2.8) can be regarded just as some kind of scattering relation among different directions at boundary. So our angular discretization plus linear interpolation for general boundary conditions inherits those nice properties for approximating the scattering term in RTE, i.e., a true averaging operation among directions. When coupled with the finite difference scheme for spatial discretization, it can be easily shown that the fully discretized linear system has an $M$-matrix even with general reflection boundary condition (see the proof for Theorem 6).

\section{ERror estimates AND CONVERGENCE ANALYSIS}

3.1. Angular discretization. The main result in this section is to show that the solution $\phi$ after angular discretization of (2.3) and RTE solution $\Phi$ to (1.1) satisfies

$$
\|\phi-\Phi\|=\sqrt{\sum_{m=1}^{M} w_{m} \int_{\Omega}\left[\phi_{m}(\vec{x})-\Phi\left(\vec{x}, \hat{s}_{m}\right)\right]^{2}}=O\left(h_{a}^{2}\right) .
$$

From now on we define the following weighted $L^{2}$ norm

$$
\|\phi\|^{2}:=\sum_{m=1}^{M} w_{m} \int_{\Omega} \phi_{m}^{2}
$$

In order to prove Theorems $1-3$, we will first prove the following estimate in $2 \mathrm{D}$ (Lemma 1) and 3D (Lemma 2)

$$
\left\|I^{h_{a}}-I\right\|_{\infty}=O\left(h_{a}^{2}\right) .
$$

Here $\|\cdot\|_{\infty}$ is with respect to the angular variable. To simplify the notation, $h$ is used to represent the angular mesh size $h_{a}$ in this section from now on.

Lemma 1. Assuming $f, \Phi \in C^{2}\left(S^{1}\right)$, the aforementioned 2D angular discretization with $I^{h}\left(\hat{s}_{m}\right)=\sum_{m^{\prime}} w_{m m^{\prime}} \Phi_{m^{\prime}}$ has the second order convergence, i.e., $\left|I\left(\hat{s}_{m}\right)-I^{h}\left(\hat{s}_{m}\right)\right|=O\left(h^{2}\right)$, for all $m$.

Proof. $\left|I-I^{h}\right| \leq|I-\tilde{I}|+\left|\tilde{I}-I^{h}\right|$, where $\tilde{I}$ is defined by (2.1). We estimate these two terms separately.

First,

$$
|I-\tilde{I}|=\left|\oint_{S^{1}}(f-\tilde{f}) \Phi+\tilde{f}(\Phi-\tilde{\Phi}) d \theta\right| \leq 2 \pi\left(\|f-\tilde{f}\|_{\infty}\|\Phi\|_{\infty}+\|\tilde{f}\|_{\infty}\|\Phi-\tilde{\Phi}\|_{\infty}\right) .
$$

Due to the piecewise linear approximation, $\|f-\tilde{f}\|_{\infty}=O\left(h^{2}\right),\|\Phi-\tilde{\Phi}\|_{\infty}=O\left(h^{2}\right)$, thus $|I-\tilde{I}|=O\left(h^{2}\right)$.

Next, observe that $1-\sum w_{m m^{\prime}}^{0}=\oint_{S^{1}}(f-\tilde{f}) d \theta=O\left(h^{2}\right)$ and $w_{m m^{\prime}}^{0}-w_{m m^{\prime}}$ has the same sign for all $m^{\prime}$ (because $w_{m m^{\prime}}^{0}$ 's are all non-negative and $w_{m m^{\prime}}$ is the normalized $w_{m m^{\prime}}^{0}$, i.e., $\left.w_{m m^{\prime}}=w_{m m^{\prime}}^{0} /\left(\sum_{m^{\prime}} w_{m m^{\prime}}^{0}\right)\right)$,

$$
\left|\tilde{I}-I^{h}\right|=\left|\sum_{m^{\prime}}\left(w_{m m^{\prime}}^{0}-w_{m m^{\prime}}\right) \Phi_{m^{\prime}}\right| \leq|| \Phi \|_{\infty}\left|\sum w_{m m^{\prime}}^{0}-1\right|=O\left(h^{2}\right) .
$$


We have proved (3.3) in 2D. In 3D, the angular discretization (Figure 2) is similar except that the weight $w_{m m^{\prime}}^{0}$ is computed on the flat element of the triangulation of the sphere with piecewise linear approximation. Then the weight $w_{m m^{\prime}}$ is normalized from $w_{m m^{\prime}}^{0}$.

Lemma 2. Assuming $f, \Phi \in C^{2}\left(S^{2}\right)$, the $3 \mathrm{D}$ angular discretization as described above with $I^{h}\left(\hat{s}_{m}\right)=\sum_{m^{\prime}} w_{m m^{\prime}} \Phi_{m^{\prime}}$ has the second order convergence, i.e., $\mid I\left(\hat{s}_{m}\right)-$ $I^{h}\left(\hat{s}_{m}\right) \mid=O\left(h^{2}\right)$, for all $m$.

Proof. Consider a triangle element of the triangulation of $S^{2}$ as depicted in Figure 2. Let $\vec{x}$ be a point on the triangle denoted by $\triangle$ and let $\vec{x}^{r}=\vec{x} /|\vec{x}|$ be the corresponding projection onto the sphere. The map is one-to-one, onto and smooth on each triangular element. In particular, $\vec{x}^{r}-\vec{x}=\vec{x}^{r}(1-|\vec{x}|)$ with $1-|\vec{x}|=O\left(h^{2}\right)$. The change of variable $\vec{x}^{r}(\vec{x})$ is smooth and

$$
\nabla_{\vec{x}} \vec{x}^{r}=\frac{1}{|\vec{x}|}\left(I-\frac{\vec{x}}{|\vec{x}|} \otimes \frac{\vec{x}}{|\vec{x}|}\right) .
$$

It can be easily seen that $I-\frac{\vec{x}}{|\vec{x}|} \otimes \frac{\vec{x}}{|\vec{x}|}$ is almost the projection to the triangle plane up to a $O(h)$ perturbation. So the corresponding surface area on the sphere and the surface area on each triangle element satisfies $d s\left(\vec{x}^{r}\right)=d s(\vec{x})\left(1+O\left(h^{2}\right)\right)$. Also the piecewise linear approximation $\tilde{f}(\vec{x})$ of any function $f\left(\vec{x}^{r}(\vec{x})\right) \in C^{2}(\triangle)$ on each triangle satisfies $\|f-\tilde{f}\|_{\infty}=O\left(h^{2}\right)$.

Therefore,

$$
I=\oint_{S^{2}} f \Phi d s\left(\vec{x}^{r}\right)=\sum_{i} \int_{\triangle_{i}}\left[\tilde{f}+O\left(h^{2}\right)\right]\left[\tilde{\Phi}+O\left(h^{2}\right)\right]\left[1+O\left(h^{2}\right)\right] d s(\vec{x})=\tilde{I}+O\left(h^{2}\right),
$$

where $\tilde{I}=\sum_{i} \int_{\triangle_{i}} \tilde{f} \tilde{\Phi} d s(\vec{x})$. In particular, $\tilde{I}\left(\hat{s}_{m}\right)=\sum_{m^{\prime}} w_{m m^{\prime}}^{0} \Phi_{m^{\prime}}$. Use the same argument as in Lemma $1,\left|\tilde{I}\left(\hat{s}_{m}\right)-I^{h}\left(\hat{s}_{m}\right)\right|=O\left(h^{2}\right)$.

Remark 3.1. H-G function (1.2) belongs to $C^{2}$ for $g<1$. When $g=1, f$ is a Delta function in angle. So there is no scattering among different directions and $I(\hat{s})=\Phi(\hat{s})$. As a result, the system (2.3) is reduced to a set of uncoupled firstorder transport equations, which can be solved easily and the analysis is the same as that for a single transport equation.

Next we will use Lemmas 1 and 2 to prove the second-order convergence (3.3) for the angular discretization in Theorem 1 . However, due to no rotation invariance for the triangulation on the angular sphere, we need the following lemma in order to proceed in 3D. For comparison, the grid is uniform and rotationally invariant on the circle in $2 \mathrm{D}$. As a result, $w_{m}$ 's are equal in $2 \mathrm{D}$, while they are not in $3 \mathrm{D}$. For the same reason, the angular weight $w_{m m^{\prime}}$ 's are symmetric in $2 \mathrm{D}$, i.e., $w_{m m^{\prime}}=w_{m^{\prime} m}$ which is not true in $3 \mathrm{D}$ either. However, this broken symmetry in $3 \mathrm{D}$ can be remedied in the proof of Theorem 3 using the following lemma between $w_{m m^{\prime}}$ and $w_{m^{\prime} m}$. Notice that although the lemma is for the normalized weight $w_{m m^{\prime}}$ 's, it also holds for $w_{m m^{\prime}}^{0}$ 's since $w_{m m^{\prime}}$ has the same dilation from $w_{m m^{\prime}}^{0}$ and $\frac{w_{m m^{\prime}}}{w_{m m^{\prime}}^{0}}=1+O\left(h^{2}\right)$ for all $m^{\prime}$.

Lemma 3. $\frac{w_{m m^{\prime}}}{w_{m^{\prime}}}-\frac{w_{m^{\prime} m}}{w_{m}}=O(h)$ for angular discretization in $3 \mathrm{D}$. 
Proof. We have

$$
\begin{aligned}
w_{m m^{\prime}}^{0}=\oint_{S^{2}} \tilde{f}\left(\hat{s}_{m} \cdot \hat{s}\right) L_{m^{\prime}}(\hat{s}) d \hat{s} & =\oint_{S^{2}}\left(\tilde{f}\left(\hat{s}_{m} \cdot \hat{s}_{m^{\prime}}\right)+O(h)\right) L_{m^{\prime}}(\hat{s}) d \hat{s} \\
& =\left(\tilde{f}\left(\hat{s}_{m} \cdot \hat{s}_{m^{\prime}}\right)+O(h)\right) w_{m^{\prime}}
\end{aligned}
$$

Similarly, $w_{m^{\prime} m}^{0}=\left(\tilde{f}\left(\hat{s}_{m^{\prime}} \cdot \hat{s}_{m}\right)+O(h)\right) w_{m}$. Therefore, $\frac{w_{m m^{\prime}}^{0}}{w_{m^{\prime}}}-\frac{w_{m^{\prime} m}^{0}}{w_{m}}=O(h)$. From the fact $\frac{w_{m m^{\prime}}}{w_{m m^{\prime}}^{0}}=1+O\left(h^{2}\right)$ for each $m^{\prime}$, the claim follows.

Theorem 1. With the angular discretization (2.3), the approximate solution $\phi$ satisfies

$$
\max _{\vec{x}, m}\left|\phi_{m}(\vec{x})-\Phi\left(\vec{x}, \hat{s}_{m}\right)\right|=O\left(h^{2}\right),
$$

if the solution $\Phi(\vec{x}, \hat{s})$ to the RTE is $C^{2}(S)$.

Proof. For the simplicity of notation, let $\Phi_{m}$ denote $\Phi\left(\vec{x}, \hat{s}_{m}\right)$,

$$
\sum_{m^{\prime}=1}^{M} w_{m m^{\prime}} \phi_{m^{\prime}}-\oint f\left(\hat{s}_{m}, \hat{s}^{\prime}\right) \Phi\left(\hat{s}^{\prime}\right) d \hat{s}^{\prime}=\sum_{m^{\prime}=1}^{M} w_{m m^{\prime}}\left(\phi_{m^{\prime}}-\Phi_{m^{\prime}}\right)+D_{m}
$$

with

$$
D_{m}:=\sum_{m^{\prime}} w_{m m^{\prime}} \Phi_{m^{\prime}}-\oint f\left(\hat{s}_{m}, \hat{s}^{\prime}\right) \Phi\left(\hat{s}^{\prime}\right) d \hat{s}^{\prime} .
$$

Let $\varepsilon_{m}=\phi_{m}-\Phi_{m}$, and then $\varepsilon_{m}$ satisfies

$$
\hat{s}_{m} \cdot \nabla \varepsilon_{m}+\mu_{t} \varepsilon_{m}=\mu_{s} \sum_{m^{\prime}=1}^{M} w_{m m^{\prime}} \varepsilon_{m^{\prime}}+D_{m} .
$$

Since $\Omega$ is a bounded closed domain, assume that $\max _{\vec{x}, m}\left|\phi_{m}(\vec{x})-\Phi\left(\vec{x}, \hat{s}_{m}\right)\right|=e_{\infty}$ is obtained for for some $\tilde{m}$ at some point $\tilde{\vec{x}}$.

(1) If $\tilde{\vec{x}}$ is in the interior of $\Omega, \nabla \varepsilon_{\tilde{m}}(\tilde{\vec{x}})=0$ and because $w_{m m^{\prime}} \geq 0, \sum_{m^{\prime}} w_{m m^{\prime}}=$ 1 , from (3.7) we have

$$
\mu_{t} e_{\infty} \leq \mu_{s} \sum_{m^{\prime}} w_{\tilde{m} m^{\prime}} e_{\infty}+\left|D_{\tilde{m}}\right| \Rightarrow \mu_{a} e_{\infty} \leq\|D\|_{\infty} \Rightarrow e_{\infty}=O\left(h^{2}\right) .
$$

(2) If $\tilde{\vec{x}}$ belongs to the boundary $\partial \Omega$,

(a) If $\left(\tilde{\vec{x}}, \hat{s}_{\tilde{m}}\right) \in \Gamma^{+}$, i.e., $\hat{s}_{\tilde{m}} \cdot \hat{n}>0$, we have $\hat{s}_{\tilde{m}} \cdot \nabla \varepsilon_{\tilde{m}}(\tilde{\vec{x}}) \geq 0$, Again from (3.7) we have $e_{\infty}=O\left(h^{2}\right)$.

(b) Otherwise if $\left(\tilde{\vec{x}}, \hat{s}_{\tilde{m}}\right) \in \Gamma^{-}$, for the general reflection boundary condition with discretization (2.8) we have

$$
\varepsilon_{\tilde{m}}(\tilde{\vec{x}})=\sum_{m^{\prime}} r_{\tilde{m} m^{\prime}} \varepsilon_{m^{\prime}}(\vec{x})+\bar{D}, \quad\left(\tilde{\vec{x}}, \hat{s}_{\tilde{m}}\right) \in \Gamma^{-},\left(\tilde{\vec{x}}, \hat{s}_{m^{\prime}}\right) \in \Gamma^{+},
$$

where $\bar{D}$ is the truncation error due to linear interpolation of the real reflection condition for at the boundary and is of order $O\left(h^{2}\right)$. Also $r_{\tilde{m} m^{\prime}} \geq 0$ and $\sum_{m^{\prime}} r_{\tilde{m} m^{\prime}} \leq 1$. Combined with the argument for (a) we have $e_{\infty}=O\left(h^{2}\right)$.

Hence we complete the proof for (3.4). 
Theorem 2 (Vacuum Boundary Condition). Assume the solution $\Phi(\vec{x}, \hat{s})$ to the $R T E$ is $C^{2}(S)$. With vacuum boundary condition and sufficiently fine angular mesh

$$
\|\phi-\Phi\|=\sqrt{\sum_{m=1}^{M} w_{m} \int_{\Omega}\left[\phi_{m}(\vec{x})-\Phi\left(\vec{x}, \hat{s}_{m}\right)\right]^{2} d \vec{x}}=O\left(h^{2}\right) .
$$

Proof. Multiplying both sides of (3.7) with $w_{m} \varepsilon_{m}$, integrating over the spatial domain and summing over all angular directions, we have

$$
\begin{aligned}
& \frac{1}{2} \sum_{m=1}^{M} w_{m} \int_{\partial \Omega} \hat{s}_{m} \cdot \hat{n} \varepsilon_{m}^{2}+\sum_{m=1}^{M} w_{m} \int_{\Omega} \mu_{t} \varepsilon_{m}^{2} \\
& =\sum_{m=1}^{M} \sum_{m^{\prime}=1}^{M} \int_{\Omega} \mu_{s} w_{m} w_{m m^{\prime}} \varepsilon_{m^{\prime}} \varepsilon_{m}+\sum_{m=1}^{M} \int_{\Omega} w_{m} D_{m} \varepsilon_{m} .
\end{aligned}
$$

With vacuum boundary condition, $\varepsilon_{m}=0$ for $\hat{s}_{m} \cdot \hat{n}<0$, so the boundary integral becomes

$$
\frac{1}{2} \sum_{m=1}^{M} w_{m} \int_{\partial \Omega} \hat{s}_{m} \cdot \hat{n} \varepsilon_{m}^{2}=\frac{1}{2} \int_{\partial \Omega} \sum_{m: \hat{s}_{m} \cdot \hat{n} \geq 0} w_{m} \hat{s}_{m} \cdot \hat{n} \varepsilon_{m}^{2} \geq 0 .
$$

Next we analyze the first term on the right-hand side of (3.9),

$$
\sum_{m, m^{\prime}=1}^{M} \int_{\Omega} \mu_{s} w_{m} w_{m m^{\prime}} \varepsilon_{m^{\prime}} \varepsilon_{m} \leq \frac{1}{2} \sum_{m, m^{\prime}=1}^{M} \int_{\Omega} \mu_{s} w_{m} w_{m m^{\prime}}\left(\varepsilon_{m^{\prime}}^{2}+\varepsilon_{m}^{2}\right) .
$$

On one hand, $\sum_{m, m^{\prime}} w_{m} w_{m m^{\prime}} \varepsilon_{m}^{2}=\sum_{m} w_{m} \varepsilon_{m}^{2}$ since $\sum_{m^{\prime}} w_{m m^{\prime}}=1$ from our angular normalization; on the other hand, using Lemma 3 ,

$$
\sum_{m, m^{\prime}} w_{m} w_{m m^{\prime}} \varepsilon_{m^{\prime}}^{2}=\sum_{m^{\prime}} \sum_{m}\left[\left(w_{m^{\prime}} w_{m^{\prime} m}+w_{m} w_{m^{\prime}} O(h)\right)\right] \varepsilon_{m^{\prime}}^{2}=\sum_{m^{\prime}} w_{m^{\prime}}(1+O(h)) \varepsilon_{m^{\prime}}^{2} .
$$

Therefore, when $h$ is small enough, we have

$$
\sum_{m, m^{\prime}} w_{m} w_{m m^{\prime}} \varepsilon_{m^{\prime}}^{2} \leq(1+2 \delta) \sum_{m^{\prime}} w_{m^{\prime}} \varepsilon_{m^{\prime}}^{2}
$$

with $\delta \sim O(h)$. Notice that $\delta=0$ in $2 \mathrm{D}$ due to rotational invariance.

Therefore, from (3.9) and (3.11) we have

$$
\sum_{m=1}^{M} w_{m} \int_{\Omega}\left(\mu_{a}-\delta \mu_{s}\right) \varepsilon_{m}^{2} \leq \sum_{m=1}^{M} w_{m} \int_{\Omega} D_{m} \varepsilon_{m} .
$$

Since $\mu_{a}$ is bounded below with a positive constant $C_{\mu}$, we have $\mu_{a}-\delta \mu_{s} \geq C_{\mu}$ in 2D with $\delta=0$, while $\mu_{a}-\delta \mu_{s} \geq C$ in $3 \mathrm{D}$ for some positive constant $C$ when $h$ is small enough. Then we apply Holder's inequality to the right-hand side of (3.12) such that $\sum_{m} w_{m} \int_{\Omega} D_{m} \varepsilon_{m} \leq\|D\| \cdot\|\varepsilon\|$.

As a result, we have

$$
\|\varepsilon\| \leq C\|D\|
$$

for some positive constant $C$. Moreover, from Lemmas 1 and 2, we have $\|D\|_{\infty}=$ $O\left(h^{2}\right)$. Thus, $\|\phi-\Phi\|=O\left(h^{2}\right)$. 
The boundary term $\sum_{m=1}^{M} w_{m} \int_{\partial \Omega} \hat{s}_{m} \cdot \hat{n} \varepsilon_{m}^{2}$ becomes more complicated for reflection boundary condition (1.3). For simplicity, let us first assume that for each incoming direction $\hat{s}_{m}$ on the angular mesh there is a corresponding outgoing direction $\hat{s}_{m^{\prime}}$ on the angular mesh with the following reflection condition

$$
\hat{s}_{m} \cdot \hat{n}=-\hat{s}_{m^{\prime}} \cdot \hat{n}, \quad \phi_{m}=r_{m m^{\prime}} \phi_{m^{\prime}}, \quad \Phi_{m}=r_{m m^{\prime}} \Phi_{m^{\prime}}, \quad 0 \leq r_{m m^{\prime}} \leq 1 .
$$

Then

$$
\sum_{m=1}^{M} w_{m} \int_{\partial \Omega} \hat{s}_{m} \cdot \hat{n} \varepsilon_{m}^{2}=\int_{\partial \Omega} \sum_{m: \hat{s}_{m} \cdot \hat{n} \geq 0}\left(w_{m}-w_{m^{\prime}} r_{m^{\prime} m}^{2}\right) \hat{s}_{m} \cdot \hat{n} \varepsilon_{m}^{2}
$$

which is analogous to the continuous case. In $2 \mathrm{D}$, each term on the right-hand side is non-negative since $w_{m}=w_{m^{\prime}}$ and $1-r_{m m^{\prime}}^{2} \geq 0$. In general, the above assumption (3.14) is not true. However, if we assume a non-perfect reflection condition, i.e., $R\left(\vec{x}, \hat{s}, \hat{s}^{\prime}\right)<1$ in (1.3), we can prove the following lemma which will be enough for the error estimate for the general reflection boundary condition in $2 \mathrm{D}$.

Lemma 4. If there is no perfect reflection at the boundary, i.e., $R\left(\vec{x}, \hat{s}, \hat{s}^{\prime}\right)<1$, $\sum_{m} r_{m m^{\prime}}<1$ is true in $2 \mathrm{D}$.

Proof. In 2D, since the angular mesh is uniform, the corresponding reflected angles are also uniform. Moreover, we use linear interpolation between two neighboring angles to interpolate the reflection condition (Figure 3), so at the boundary $\partial \Omega$, we have

$$
\sum_{m} r_{m m^{\prime}}=\alpha_{m_{1}^{\prime} m^{\prime}} R\left(\hat{s}_{m_{1}}, \hat{s}_{m_{1}^{\prime}}\right)+\alpha_{m_{2}^{\prime} m^{\prime}} R\left(\hat{s}_{m_{2}}, \hat{s}_{m_{2}^{\prime}}\right), \quad \alpha_{m_{1}^{\prime} m^{\prime}}+\alpha_{m_{2}^{\prime} m^{\prime}}=1
$$

where $\hat{s}_{m^{\prime}}$ is an outgoing direction that contributes to both incoming directions $\hat{s}_{m_{1}}$ and $\hat{s}_{m_{2}}$ in the linear interpolation and $\alpha_{m_{1}^{\prime} m^{\prime}}, \alpha_{m_{2}^{\prime} m^{\prime}}$ are the linear weights. From the non-perfect reflection condition, we have $\sum_{m} r_{m m^{\prime}}<\sigma<1$.

Next we prove the similar $L_{2}$ error estimate for general reflection boundary condition in 2D.

Theorem 3 (Reflection Boundary Condition). In 2D, assume the solution $\Phi(\vec{x}, \hat{s})$ to the RTE is $C^{2}\left(S^{1}\right)$. With no perfect reflection at the boundary, i.e., $R\left(\vec{x}, \hat{s}, \hat{s}^{\prime}\right)<$ $\sigma<1$ in (1.3), and if the angular mesh is fine enough,

$$
\|\phi-\Phi\|=O\left(h^{2}\right)
$$

Proof. The key is to control the boundary term $\sum_{m=1}^{M} w_{m} \int \hat{s}_{m} \cdot \hat{n} \varepsilon_{m}^{2} d \Gamma$ in (3.9). For the general reflection boundary condition (1.3) approximated by linear interpolation (2.8), we have

$$
\varepsilon_{m}(\vec{x})=\sum_{m^{\prime}} r_{m m^{\prime}}(\vec{x}) \varepsilon_{m^{\prime}}(\vec{x})+\bar{D}_{m}(\vec{x}), \quad\left(\vec{x}, \hat{s}_{m}\right) \in \Gamma^{-},\left(\vec{x}, \hat{s}_{m^{\prime}}\right) \in \Gamma^{+},
$$


where $\bar{D}_{m}(\vec{x})$ is the truncation error due to linear interpolation of the real reflection condition for at the boundary and is of order $O\left(h^{2}\right)$.

$$
\begin{aligned}
& \sum_{m=1}^{M} w_{m} \int_{\partial \Omega} \hat{s}_{m} \cdot \hat{n} \varepsilon_{m}^{2}=\sum_{\hat{s}_{m} \cdot \hat{n} \geq 0} w_{m} \int_{\partial \Omega}\left|\hat{s}_{m} \cdot \hat{n}\right| \varepsilon_{m}^{2} \\
& \quad-\sum_{\hat{s}_{m} \cdot \hat{n} \leq 0} w_{m} \int_{\partial \Omega}\left|\hat{s}_{m} \cdot \hat{n}\right| \varepsilon_{m}^{2}=\sum_{\hat{s}_{m} \cdot \hat{n} \geq 0} w_{m} \int_{\partial \Omega}\left|\hat{s}_{m} \cdot \hat{n}\right| \varepsilon_{m}^{2} \\
& \quad-\sum_{\hat{s}_{m} \cdot \hat{n} \leq 0} w_{m} \int_{\partial \Omega}\left|\hat{s}_{m} \cdot \hat{n}\right|\left(\sum_{\hat{s}_{m^{\prime}} \cdot \hat{n} \geq 0} r_{m m^{\prime}} \varepsilon_{m^{\prime}}+\bar{D}_{m}\right)^{2}
\end{aligned}
$$

Now let us estimate the second term in the above expression:

$$
\begin{aligned}
& \sum_{\hat{s}_{m} \cdot \hat{n} \leq 0} w_{m} \int_{\partial \Omega}\left|\hat{s}_{m} \cdot \hat{n}\right|\left(\sum_{\hat{s}_{m^{\prime}} \cdot \hat{n} \geq 0} r_{m m^{\prime}} \varepsilon_{m^{\prime}}+\bar{D}_{m}\right)^{2} \\
& \leq \sum_{\hat{s}_{m} \cdot \hat{n} \leq 0} w_{m} \int_{\partial \Omega}\left|\hat{s}_{m} \cdot \hat{n}\right|\left[\frac{1}{\sigma}\left(\sum_{\hat{s}_{m^{\prime}} \cdot \hat{n} \geq 0} r_{m m^{\prime}} \varepsilon_{m^{\prime}}\right)^{2}+\frac{1}{1-\sigma} \bar{D}_{m}^{2}\right] \\
& \leq \sum_{\hat{s}_{m} \cdot \hat{n} \leq 0} w_{m} \int_{\partial \Omega}\left|\hat{s}_{m} \cdot \hat{n}\right|\left[\sum_{\hat{s}_{m^{\prime}} \cdot \hat{n} \geq 0} r_{m m^{\prime}} \varepsilon_{m^{\prime}}^{2}+\frac{1}{1-\sigma} \bar{D}_{m}^{2}\right] \\
& \leq \sum_{\hat{s}_{m^{\prime}} \cdot \hat{n} \geq 0} w_{m^{\prime}} \int_{\partial \Omega} \varepsilon_{m^{\prime}}^{2}\left(\sum_{\hat{s}_{m} \cdot \hat{n} \leq 0} r_{m m^{\prime}}\left|\hat{s}_{m} \cdot \hat{n}\right|\right) \\
& \quad \quad \frac{1}{1-\sigma} \sum_{\hat{s}_{m} \cdot \hat{n} \leq 0} w_{m} \int_{\partial \Omega}\left|\hat{s}_{m} \cdot \hat{n}\right| \bar{D}_{m}^{2} \\
& =\sum_{\hat{s}_{m^{\prime}} \cdot \hat{n} \geq 0} w_{m^{\prime}} \int_{\partial \Omega} \varepsilon_{m^{\prime}}^{2}\left(\alpha_{m_{1}^{\prime} m^{\prime}} R_{m_{1} m_{1}^{\prime}}\left|\hat{s}_{m_{1}^{\prime}} \cdot \hat{n}\right|+\alpha_{m_{2}^{\prime} m^{\prime}} R_{m_{2} m_{2}^{\prime}}\left|\hat{s}_{m_{2}^{\prime}} \cdot \hat{n}\right|\right) \\
& \quad \quad+\frac{1}{1-\sigma} \sum_{\hat{s}_{m} \cdot \hat{n} \leq 0} w_{m} \int_{\partial \Omega}\left|\hat{s}_{m} \cdot \hat{n}\right| \bar{D}_{m}^{2} \\
& =\sum_{\hat{s}_{m^{\prime}} \cdot \hat{n} \geq 0} w_{m^{\prime}} \int_{\partial \Omega} \varepsilon_{m^{\prime}}^{2}\left(O\left(h^{2}\right)+R_{m m^{\prime}}\left|\hat{s}_{m^{\prime}} \cdot \hat{n}\right|\right) \\
& \quad+\frac{1}{1-\sigma} \sum_{\hat{s}_{m} \cdot \hat{n} \leq 0} w_{m} \int_{\partial \Omega}\left|\hat{s}_{m} \cdot \hat{n}\right| \bar{D}_{m}^{2} \\
& \leq \sum_{\hat{s}_{m^{\prime}} \cdot \hat{n} \geq 0} w_{m^{\prime}} \int_{\partial \Omega}(1+O(h)) \sigma\left|\hat{s}_{m^{\prime}} \cdot \hat{n}\right| \varepsilon_{m^{\prime}}^{2}+\frac{1}{1-\sigma} \sum_{\hat{s}_{m} \cdot \hat{n} \leq 0} w_{m} \int_{\partial \Omega}\left|\hat{s}_{m} \cdot \hat{n}\right| \bar{D}_{m}^{2},
\end{aligned}
$$

where $R_{m m^{\prime}}:=R\left(\vec{x}, \hat{s}_{m}, \hat{s}_{m^{\prime}}\right)$, the second inequality uses Jensen's inequality due to the convexity of the function $f(x)=x^{2}$, the second equality is due to linear interpolation of the smooth reflection function, i.e., $\alpha_{m_{1}^{\prime} m^{\prime}}+\alpha_{m_{2}^{\prime} m^{\prime}}=1$ as shown in Figure 3, $w_{m}$ 's are all equal, and $\sum_{m} r_{m m^{\prime}}<\sigma$ from Lemma 4 . When $h$ is small enough we can find $\sigma<\sigma^{\prime}<1$ such that

$$
\begin{aligned}
\sum_{m=1}^{M} w_{m} \int_{\partial \Omega} \hat{s}_{m} \cdot \hat{n} \varepsilon_{m}^{2} \geq(1 & \left.-\sigma^{\prime}\right) \sum_{\hat{s}_{m} \cdot \hat{n} \geq 0} w_{m} \int_{\partial \Omega}\left|\hat{s}_{m} \cdot \hat{n}\right| \varepsilon_{m}^{2} \\
& -\frac{1}{1-\sigma} \sum_{\hat{s}_{m} \cdot \hat{n} \leq 0} w_{m} \int_{\partial \Omega}\left|\hat{s}_{m} \cdot \hat{n}\right| \bar{D}_{m}^{2}
\end{aligned}
$$

From (3.12) we have

Combining the above results we get

$$
\frac{C}{2}\|\varepsilon\|^{2} \leq \frac{1}{2 C}\|D\|^{2} .
$$

$\left(1-\sigma^{\prime}\right) \sum_{\hat{s}_{m} \cdot \hat{n} \geq 0} w_{m} \int_{\partial \Omega}\left|\hat{s}_{m} \cdot \hat{n}\right| \varepsilon_{m}^{2}+C\|\varepsilon\|^{2} \leq \frac{1}{1-\sigma} \sum_{\hat{s}_{m} \cdot \hat{n} \leq 0} w_{m} \int_{\partial \Omega}\left|\hat{s}_{m} \cdot \hat{n}\right| \bar{D}_{m}^{2}+\frac{1}{C}\|D\|^{2}$.

Since both truncation errors in angular discretization for boundary condition and scattering operator are of order $O\left(h^{2}\right)$, we have proved the result.

The complication for reflection boundary condition in 3D mainly comes from: (1) the difficulty for obtaining the similar inequality as (2.9) in some pathological case when $\hat{s}_{m}$ is almost tangential to the boundary at $\vec{x}$, and (2) the non-uniformity of the angular mesh, i.e., the failure for establishing the similar claim in Lemma 4. 
3.2. Spatial discretization by DG. In this section, we give an error estimate for the spatial DG discretization of coupled transport equations (2.3). First we give a brief summary on DG method and its analysis on the following single transport equation on a bounded domain $\Omega$ with boundary condition on inflow part of the boundary $\partial \Omega_{-}=\{\vec{x} \in \partial \Omega: \hat{n}(\vec{x}) \cdot \hat{s}<0\}$,

$$
\begin{cases}\hat{s} \cdot \nabla u(\vec{x})+c(\vec{x}) u(\vec{x})=f(\vec{x}) & \text { in } \Omega, \\ u=g & \text { on } \partial \Omega_{-},\end{cases}
$$

where $\hat{n}(\vec{x})$ is the outward unit normal to $\partial \Omega$ at $\vec{x}$.

People have been studying the DG method for the convection equation (3.18) since 1974 with the first error estimate on general meshes [7], i.e.,

$$
\left\|u-u_{h}\right\|_{2} \leq C h^{k}|u|_{H^{k+1}}
$$

followed by the improved convergence result [2] in 1986 with

$$
\left\|u-u_{h}\right\|_{2} \leq C h^{k+1 / 2}|u|_{H^{k+1}},
$$

where $k$ is the degree of polynomial approximation in each element.

Actually, the result was proved with a stronger norm than $L^{2}$ norm. Besides, $L^{p}$ stability and error estimates for $p \neq 2$ were analyzed for piecewise linear DG on uniform or piecewise uniform triangulation [2]. However, since the sign of $c(\vec{x})$ is undetermined, a special auxiliary function has to be introduced to control the possible exponential growth and get the error estimate. The construction of such an auxiliary function requires convexity of the domain $\Omega$. Two years later, assuming the conforming triangulations that are obtained with slabs of parallelograms divided into two triangles, [3] showed an optimal convergence estimate

$$
\left\|u-u_{h}\right\|_{2} \leq C h^{k+1}|u|_{H^{k+2}} .
$$

Recently, 1] proved a similar result

$$
\left\|u-u_{h}\right\|_{2} \leq C h^{k+1}|u|_{H^{k+1}}
$$

for meshes that are not necessarily conforming or subject to any uniformity condition, however, made of simplexes of a unique outflow face.

Although we need to deal with a system of coupled transport equations (2.3), the coupling is not very strong. In particular, due to the absorption, $\mu_{t}-\mu_{s}=\mu_{a}>0$, which is also a crucial condition for the stability of the original RTE (1.1), our proof is relatively simple.

For notation convenience, we use $h\left(h_{a}\right)$ to represent the spatial (angular) mesh size hereafter. Let us start with the DG formulation of RTE (2.3) with vacuum boundary condition. Given a triangulation $T_{h}$ with $h=\sup _{K \in T_{h}} \operatorname{diam}(K)$ and the finite-dimensional space $V_{h}^{k}$ which is composed of functions that are polynomials of at most $k$ degrees on each $K \in T_{h}$, let $(u, v)_{K}=\int_{K} u v$ and $\langle u, v\rangle_{B}=\int_{B} u v \mid \hat{s}$. $\hat{n} \mid$ with $\|u\|_{K}^{2}=(u, u)_{K}$ and $\langle\langle u\rangle\rangle_{B}^{2}=\langle u, u\rangle_{B}$, where $B \subset \Gamma_{h} \bigcup \partial \Omega$ with $\Gamma_{h}=$ $\left(\bigcup_{k \in T_{h}} \partial K\right) \backslash \partial \Omega$. For each angular direction $m$, let $\partial \Omega_{-(+)}^{m}=\left\{\vec{x} \in \partial \Omega: \hat{n}(\vec{x}) \cdot \hat{s}_{m} \leq\right.$ $(>) 0\}$. Then DG approximation $\phi^{h} \in\left(V_{h}^{k}\right)^{M}$ of the solution of (2.3) satisfies

$$
A_{m}\left(\phi_{m}^{h}, v\right)=(q, v)+\left\langle q_{b}, v\right\rangle_{\partial \Omega_{-}^{m}}, \quad \text { for all } v \in V_{h}^{k}, \text { for each } m,
$$


where

$$
\begin{gathered}
A_{m}\left(\phi_{m}^{h}, v\right)=\sum_{K}\left(\hat{s}_{m} \cdot \nabla \phi_{m}^{h}+\mu_{t} \phi_{m}^{h}-\mu_{s} \sum_{m^{\prime}} w_{m m^{\prime}} \phi_{m^{\prime}}^{h}, v\right)_{K} \\
+\left\langle\phi_{m}^{h+}-\phi_{m}^{h-}, v^{+}\right\rangle_{\Gamma_{h}^{m}}+\left\langle\phi_{m}^{h}, v\right\rangle_{\partial \Omega_{-}^{m}}
\end{gathered}
$$

with $\phi_{m}^{h \pm}=\lim _{\epsilon \rightarrow 0^{ \pm}} \phi_{m}^{h}\left(\vec{x} \pm \epsilon \hat{s}_{m}\right)$ and $\langle u, v\rangle_{\Gamma_{h}^{m}}=\int_{\Gamma_{h}} u v\left|\hat{s}_{m} \cdot \hat{n}\right|$. Here $q$ and $q_{b}$ represent the internal and boundary source respectively. Note that we can replace $\phi^{h}$ by the exact solution $\phi$ in (3.23). That is we have the following consistency relation

$$
A_{m}\left(\phi_{m}-\phi_{m}^{h}, v\right)=0, \quad \text { for all } v \in V_{h}^{k}, \text { for each } m,
$$

Notice that (3.23) can be regarded as a system of (3.18) coupled by the scattering term. Therefore, (3.23) can be analyzed similarly as the vector form of (3.18) with non-negative $c$, once we can control the scattering term. This is exactly how it will be carried out in the proof for Theorem 4. Here we assume the shape-regularity condition on the mesh, i.e., there is a constant $\sigma>0$ such that $h_{K} / \rho_{K} \geq \sigma$ for each simplex $K \in T_{h}$, where $h_{K}$ is the diameter of the simplex $K$ and $\rho_{k}$ is the diameter of the largest ball included in $K$.

Theorem 4 (Vacuum Boundary Condition). With vacuum boundary condition, when the triangulation $T_{h}$ satisfies the shape-regularity condition and $h, h_{a}$ is small enough, the error between DG solution $\phi^{h}$ via (3.23) and the exact solution $\phi$ of (2.3) with inflow boundary condition satisfies

$$
\| \phi-\phi^{h}|| \leq C h^{k+1 / 2}|\phi|_{k+1},
$$

where the norm $\|\cdot\|$ is defined by (3.2) and $|\phi|_{k+1}^{2}:=\sum_{m=1}^{M} w_{m}\left|\phi_{m}\right|_{H^{k+1}}^{2}$.

Proof. Let $\tilde{\phi}_{m}$ be the $L_{2}$ projection of $\phi_{m}$ into $V_{h}$, i.e.,

$$
\left(\phi_{m}-\tilde{\phi}_{m}, v\right)_{K}=0, v \in V_{h}^{k} \text {. }
$$

Then let us consider (3.23) for $e=\phi-\phi^{h}$. Using the consistency relation (3.25), we have the following with $\tilde{e}=\phi-\tilde{\phi}$,

$$
A_{m}\left(e_{m}, e_{m}\right)=A_{m}\left(e_{m}, \tilde{e}_{m}\right) .
$$

We first estimate $A_{m}\left(e_{m}, e_{m}\right)$. Since

$$
\left(\hat{s}_{m} \cdot \nabla e_{m}, e_{m}\right)_{K}=-\left(e_{m}, \hat{s}_{m} \cdot \nabla e_{m}\right)_{K}+\int_{\partial K} \hat{s}_{m} \cdot \hat{n} e_{m}^{2},
$$

we have

$$
\sum_{K}\left(\hat{s}_{m} \cdot \nabla e_{m}, e_{m}\right)_{K}=-\frac{1}{2}\left\langle e_{m}^{+}+e_{m}^{-}, e_{m}^{+}-e_{m}^{-}\right\rangle_{\Gamma_{h}^{m}}-\frac{1}{2}\left\langle\left\langle e_{m}\right\rangle\right\rangle_{\partial \Omega_{-}^{m}}^{2}+\frac{1}{2}\left\langle\left\langle e_{m}\right\rangle\right\rangle_{\partial \Omega_{+}^{m}}^{2} .
$$

As a result,

$$
\begin{gathered}
\sum_{K}\left(\hat{s}_{m} \cdot \nabla e_{m}, e_{m}\right)_{K}+\left\langle e_{m}^{+}-e_{m}^{-}, e_{m}^{+}\right\rangle_{\Gamma_{h}^{m}}+\left\langle\left\langle e_{m}\right\rangle\right\rangle_{\partial \Omega_{-}^{m}}^{2} \\
=\frac{1}{2}\left\langle\left\langle e_{m}^{+}-e_{m}^{-}\right\rangle\right\rangle_{\Gamma_{h}^{m}}^{2}+\frac{1}{2}\left\langle\left\langle e_{m}\right\rangle\right\rangle_{\partial \Omega^{m}}^{2},
\end{gathered}
$$

where $\langle u, v\rangle_{\partial \Omega^{m}}=\int_{\partial \Omega} u v\left|\hat{s}_{m} \cdot \hat{n}\right|$. 
The scattering term can be estimated as follows:

$$
\begin{aligned}
& J:=\sum_{m} w_{m} \sum_{K}\left(\mu_{t} e_{m}-\mu_{s} \sum_{m^{\prime}} w_{m m^{\prime}} e_{m^{\prime}}, e_{m}\right)_{K} \\
& \geq \sum_{m} w_{m}\left[\int_{\Omega} \mu_{t} e_{m}^{2}-\frac{1}{2} \sum_{m^{\prime}} w_{m m^{\prime}} \int_{\Omega} \mu_{s}\left(e_{m^{\prime}}^{2}+e_{m}^{2}\right)\right] .
\end{aligned}
$$

On one hand,

$$
\sum_{m} w_{m} \sum_{m^{\prime}} w_{m m^{\prime}} \int_{\Omega} \mu_{s} e_{m}^{2}=\sum_{m} w_{m} \int_{\Omega} \mu_{s} e_{m}^{2} .
$$

On the other hand, using Lemma 3,

$$
\begin{aligned}
& T:=\sum_{m} w_{m} \sum_{m^{\prime}} w_{m m^{\prime}} \int_{\Omega} \mu_{s} e_{m^{\prime}}^{2} \\
& =\sum_{m} \sum_{m^{\prime}}\left(w_{m^{\prime}} w_{m^{\prime} m}+w_{m^{\prime}} w_{m} O\left(h_{a}\right)\right) \int_{\Omega} \mu_{s} e_{m^{\prime}}^{2} \\
& \leq \sum_{m^{\prime}} w_{m^{\prime}} \int_{\Omega} \mu_{s} e_{m^{\prime}}^{2}\left(1+O\left(h_{a}\right)\right) .
\end{aligned}
$$

For any $h_{a}$, there exists a constant $\delta=O\left(h_{a}\right)$ such that

$$
T \leq(1+2 \delta) \sum_{m} w_{m} \int_{\Omega} \mu_{s} e_{m}^{2}
$$

Combining (3.30) to (3.33), we have

$$
J \geq C\|e\|^{2}
$$

where $C=C_{\mu}-\delta \mid\left\|\mu_{s}\right\|_{\infty}$ with lower bound $C_{\mu}$ of $\mu_{a}$, and is non-negative when $h_{a}$ is small enough in $3 \mathrm{D}$. Notice that $C=C_{\mu}$ in $2 \mathrm{D}$ due to rotation invariance of the angular mesh. Therefore,

$$
\sum_{m} w_{m} A_{m}\left(e_{m}, e_{m}\right) \geq D\left[\|e\|^{2}+\sum_{m} w_{m}\left(\left\langle\left\langle e_{m}^{+}-e_{m}^{-}\right\rangle\right\rangle_{\Gamma_{h}^{m}}^{2}+\left\langle\left\langle e_{m}\right\rangle\right\rangle_{\partial \Omega^{m}}^{2}\right)\right],
$$

where $D=\min \left(C, \frac{1}{2}\right)$.

Next, let us estimate $A_{m}\left(e_{m}, \tilde{e}_{m}\right)$, and it goes as follows. For the boundary term, we have

$$
\begin{aligned}
& \left\langle e_{m}^{+}-e_{m}^{-}, \tilde{e}_{m}^{+}\right\rangle_{\Gamma_{h}^{m}}+\left\langle e_{m}, \tilde{e}_{m}\right\rangle_{\partial \Omega_{-}^{m}} \\
& \leq \frac{D}{4}\left(\left\langle\left\langle e_{m}^{+}-e_{m}^{-}\right\rangle\right\rangle_{\Gamma_{h}^{m}}^{2}+\left\langle\left\langle e_{m}\right\rangle\right\rangle_{\partial \Omega^{m}}^{2}\right)+\frac{1}{D}\left(\left\langle\left\langle\tilde{e}_{m}^{+}\right\rangle\right\rangle_{\Gamma_{h}^{m}}^{2}+\left\langle\left\langle\tilde{e}_{m}\right\rangle\right\rangle_{\partial \Omega^{m}}^{2}\right) .
\end{aligned}
$$

For the convection term, using the property (3.27) for the $L 2$ projection,

$$
\left(\hat{s}_{m} \cdot \nabla e_{m}, \tilde{e}_{m}\right)_{K}=\left(\hat{s}_{m} \cdot \nabla \tilde{e}_{m}, \tilde{e}_{m}\right)_{K}=\frac{1}{2} \int_{\partial K} \hat{s}_{m} \cdot \hat{n} \tilde{e}_{m}^{2} .
$$

Similarly, as before, the scattering term can be estimated as follows:

$$
\begin{aligned}
& \sum_{m} w_{m} \sum_{K}\left(\mu_{t} e_{m}-\mu_{s} \sum_{m^{\prime}} w_{m m^{\prime}} e_{m^{\prime}}, \tilde{e}_{m}\right)_{K} \\
& \quad \leq \sum_{m} w_{m}\left[\sum_{K}\left(\frac{D}{8}\left\|e_{m}\right\|_{K}^{2}+\frac{2\left\|\mu_{t}\right\|_{\infty}^{2}}{D}\left\|\tilde{e}_{m}\right\|_{K}^{2}\right)\right] \\
& \quad+\sum_{m^{\prime}} w_{m^{\prime}}\left[\sum_{K}\left(\frac{D}{8}\left\|e_{m^{\prime}}\right\|_{K}^{2}+\frac{2\left\|\mu_{s}\right\|_{\infty}^{2}}{D}\left\|\sum_{m} w_{m^{\prime} m} \tilde{e}_{m}\right\|_{K}^{2}\right)\right] \\
& \leq \frac{D}{4}\|e\|^{2}+C\|\tilde{e}\|^{2} .
\end{aligned}
$$

Last, from the standard estimates for the $L 2$ projection, we have

$$
\begin{aligned}
& \left\|\tilde{e}_{m}\right\|_{K} \leq C h_{K}^{k+1}\left|\phi_{m}\right|_{H^{k+1}}(K), \\
& \left\|\tilde{e}_{m}\right\|_{\partial K} \leq C h_{K}^{k+1 / 2}\left|\phi_{m}\right|_{H^{k+1}}(K) .
\end{aligned}
$$


With the shape-regularity assumption, from (3.36) to (3.39), we have

$$
\begin{aligned}
\sum_{m} w_{m} A_{m}\left(e_{m}, \tilde{e}_{m}\right) \leq & \frac{D}{4}\left[\|e\|^{2}+\sum_{m} w_{m}\left(\left\langle\left\langle e_{m}^{+}-e_{m}^{-}\right\rangle\right\rangle_{\Gamma_{h}^{m}}^{2}+\left\langle\left\langle e_{m}\right\rangle\right\rangle_{\partial \Omega^{m}}^{2}\right)\right] \\
& +C h^{2 k+1}|\phi|_{k+1}^{2} .
\end{aligned}
$$

Combining (3.28), (3.35) and (3.40), we have shown that

$$
\|e\| \leq C h^{k+1 / 2}|\phi|_{k+1} .
$$

In the proof $C$ always denotes some constant.

Remark 3.2. Combining estimate (3.1) for angular discretization and (3.26) with $k=1$ for our piecewise linear DG spatial discretization, we have the following estimate for our scheme:

$$
\left\|\Phi-\phi^{h}\right\|=O\left(h_{a}^{2}\right)+O\left(h^{3 / 2}\right) .
$$

However, second order accuracy is observed in both angle and space in the numerical tests in [4].

Remark 3.3. The proofs with $O\left(h^{k+1}\right)$ convergence in [1, 3] do not apply here since the required property on the spatial mesh in general is not satisfied for RTE with all possible angular directions.

For general reflection boundary condition (2.8), the DG formulation (3.24) becomes

$$
\begin{aligned}
& A_{m}\left(\phi_{m}^{h}, v\right)=\sum_{K}\left(\hat{s}_{m} \cdot \nabla \phi_{m}^{h}+\mu_{t} \phi_{m}^{h}-\mu_{s} \sum_{m^{\prime}} w_{m m^{\prime}} \phi_{m^{\prime}}^{h}, v\right)_{K} \\
& +\left\langle\phi_{m}^{h+}-\phi_{m}^{h}, v^{+}\right\rangle_{\Gamma_{h}^{m}}+\left\langle\phi_{m}^{h}-\sum_{m^{\prime}} r_{m m^{\prime}} \phi_{m^{\prime}}, v\right\rangle_{\partial \Omega_{-}^{m}}
\end{aligned}
$$

where $\sum_{m^{\prime}} r_{m m^{\prime}}=R\left(\hat{s}_{m}, \hat{s}^{\prime}\right) \leq 1$ and $\hat{s}_{m^{\prime}}$ 's are the neighboring directions used in the linear approximation to approximate the outgoing direction $\hat{s}^{\prime}$ that is reflected into the incoming direction $\hat{s}_{m}$. Now let us prove the same result as in Theorem 4 for general reflection boundary condition in $2 \mathrm{D}$.

Theorem 5 (Reflection Boundary Condition). In 2D, assume that the triangulation $T_{h}$ satisfies the shape-regularity condition and $h, h_{a}$ is small enough, the error between DG solution $\phi^{h}$ via (3.23) and the exact solution $\phi$ of (2.3) with reflection boundary condition (2.8) satisfies

$$
\| \phi-\phi^{h}|| \leq C h^{k+1 / 2}|\phi|_{k+1},
$$

if $R\left(\vec{x}, \hat{s}, \hat{s}^{\prime}\right)<\sigma<1$ (no perfect reflection), where the norm $\|\cdot\|$ is defined by (3.2) and $|\phi|_{k+1}^{2}:=\sum_{m=1}^{M} w_{m}\left|\phi_{m}\right|_{H^{k+1}}^{2}$.

Proof. Following the same computation, (3.29) becomes

$$
\begin{aligned}
& \sum_{K}\left(\hat{s}_{m} \cdot \nabla e_{m}, e_{m}\right)_{K}+\left\langle e_{m}^{+}-e_{m}^{-}, e_{m}^{+}\right\rangle_{\Gamma_{h}^{m}}+\left\langle e_{m}-\sum_{m^{\prime}} r_{m m^{\prime}} e_{m^{\prime}}, e_{m}\right\rangle_{\partial \Omega_{-}^{m}} \\
& =\frac{1}{2}\left\langle\left\langle e_{m}^{+}-e_{m}^{-}\right\rangle\right\rangle_{\Gamma_{h}^{m}}^{2}+\frac{1}{2}\left\langle\left\langle e_{m}\right\rangle\right\rangle_{\partial \Omega^{m}}^{2}-\left\langle\sum_{m^{\prime}} r_{m m^{\prime}} e_{m^{\prime}}, e_{m}\right\rangle_{\partial \Omega_{-}^{m}} .
\end{aligned}
$$

Now we estimate the last term in the above expression,

$$
\left\langle\sum_{m^{\prime}} r_{m m^{\prime}} e_{m^{\prime}}, e_{m}\right\rangle_{\partial \Omega_{-}^{m}} \leq \frac{1}{2} \int_{\partial \Omega_{-}^{m}}\left(\sum_{m^{\prime}} r_{m m^{\prime}} e_{m^{\prime}}^{2}+\sum_{m^{\prime}} r_{m m^{\prime}} e_{m}^{2}\right)\left|\hat{s}_{m} \cdot \hat{n}\right| .
$$


Following the same argument in the proof of Theorem 3, when $h_{a}$ is small enough, there exists a constant $\sigma<\sigma^{\prime}<1$ such that

$$
\sum_{m} r_{m m^{\prime}}\left|\hat{s}_{m} \cdot \hat{n}\right| \leq \sigma^{\prime}\left|\hat{s}_{m^{\prime}} \cdot \hat{n}\right| .
$$

At a point of the boundary where $\hat{n}$ is well defined, if we sum up all angles that satisfy $\hat{s}_{m} \cdot \hat{n} \leq 0$ in the above expression, $\hat{s}_{m^{\prime}}$ will go over all angles $\hat{s}_{m^{\prime}} \cdot \hat{n} \geq 0$. We have

$$
\begin{aligned}
& \sum_{m: \hat{s}_{m} \cdot \hat{n} \leq 0} w_{m}\left(\sum_{m^{\prime}} r_{m m^{\prime}} e_{m^{\prime}}^{2}+\sum_{m^{\prime}} r_{m m^{\prime}} e_{m}^{2}\right)\left|\hat{s}_{m} \cdot \hat{n}\right| \\
& \leq \sigma^{\prime} \sum_{m^{\prime}: \hat{s}_{m^{\prime}} \cdot \hat{n} \geq 0} w_{m^{\prime}} e_{m^{\prime}}^{2}\left|\hat{s}_{m^{\prime}} \cdot \hat{n}\right|+\sigma \sum_{m: \hat{s}_{m} \cdot \hat{n} \leq 0} w_{m} e_{m}^{2}\left|\hat{s}_{m} \cdot \hat{n}\right| \\
& \leq \sigma^{\prime} \sum_{m} w_{m} e_{m}^{2}\left|\hat{s}_{m} \cdot \hat{n}\right| .
\end{aligned}
$$

Therefore,

$$
\sum_{m} w_{m}\left\langle\sum_{m^{\prime}} r_{m m^{\prime}} e_{m^{\prime}}, e_{m}\right\rangle_{\partial \Omega_{-}^{m}} \leq \frac{1}{2} \sigma^{\prime} \sum_{m} w_{m}\left\langle\left\langle e_{m}\right\rangle\right\rangle_{\partial \Omega^{m}}^{2} .
$$

Hence we get an estimate similar to (3.35).

$$
\sum_{m} w_{m} A_{m}\left(e_{m}, e_{m}\right) \geq D\left[\|e\|^{2}+\sum_{m} w_{m}\left(\left\langle\left\langle e_{m}^{+}-e_{m}^{-}\right\rangle\right\rangle_{\Gamma_{h}^{m}}^{2}+\frac{1-\sigma^{\prime}}{2}\left\langle\left\langle e_{m}\right\rangle\right\rangle_{\partial \Omega^{m}}^{2}\right)\right],
$$

Now we estimate for $A_{m}\left(e_{m}, \tilde{e}_{m}\right)$. Again, the only complication comes from the boundary term. We have

$$
\left\langle e_{m}-\sum_{m^{\prime}} r_{m m^{\prime}} e_{m^{\prime}}, \tilde{e}_{m}\right\rangle_{\partial \Omega_{-}^{m}}=\left\langle e_{m}, \tilde{e}_{m}\right\rangle_{\partial \Omega_{-}^{m}}-\left\langle\sum_{m^{\prime}} r_{m m^{\prime}} e_{m^{\prime}}, \tilde{e}_{m}\right\rangle_{\partial \Omega_{-}^{m}} .
$$

For the first term, we have

$$
\left\langle e_{m}, \tilde{e}_{m}\right\rangle_{\partial \Omega_{-}^{m}} \leq \frac{D\left(1-\sigma^{\prime}\right)}{16}\left\langle\left\langle e_{m}\right\rangle\right\rangle_{\partial \Omega^{m}}^{2}+\frac{4}{D\left(1-\sigma^{\prime}\right)}\left\langle\left\langle\tilde{e}_{m}\right\rangle\right\rangle_{\partial \Omega^{m}}^{2} .
$$

To estimate the second term, we first estimate pointwisely with respect to the angular variable

$$
\begin{aligned}
& \sum_{m: \hat{s}_{m} \cdot \hat{n} \leq 0} w_{m}\left(\sum_{m^{\prime}: \hat{s}_{m^{\prime}} \cdot \hat{n} \geq 0} r_{m m^{\prime}} e_{m^{\prime}}\right) \tilde{e}_{m}\left|\hat{s}_{m} \cdot \hat{n}\right| \\
& \leq \sum_{m} w_{m}\left|\hat{s}_{m} \cdot \hat{n}\right| \sum_{m^{\prime}} r_{m m^{\prime}}\left(\frac{D\left(1-\sigma^{\prime}\right)}{32} e_{m^{\prime}}^{2}+\frac{8}{D\left(1-\sigma^{\prime}\right)} \tilde{e}_{m}^{2}\right) \\
& \leq \frac{D\left(1-\sigma^{\prime}\right)}{16} \sum_{m^{\prime}: \hat{s}_{m^{\prime}} \cdot \hat{n} \geq 0} w_{m^{\prime}}\left|\hat{s}_{m^{\prime}} \cdot \hat{n}\right| e_{m^{\prime}}^{2}+\frac{8}{D\left(1-\sigma^{\prime}\right)} \sum_{m: \hat{s}_{m} \cdot \hat{n} \leq 0} w_{m}\left|\hat{s}_{m} \cdot \hat{n}\right| \tilde{e}_{m}^{2},
\end{aligned}
$$

when the angular mesh is fine enough.

Therefore,

$$
\sum_{m} w_{m}\left\langle\sum_{m^{\prime}} r_{m m^{\prime}} e_{m^{\prime}}, \tilde{e}_{m}\right\rangle_{\partial \Omega_{-}^{m}} \leq \frac{\frac{D\left(1-\sigma^{\prime}\right)}{16} \sum_{m} w_{m}\left\langle\left\langle e_{m}\right\rangle\right\rangle_{\partial \Omega^{m}}^{2}}{+\frac{8}{D\left(1-\sigma^{\prime}\right)} \sum_{m} w_{m}\left\langle\left\langle\tilde{e}_{m}\right\rangle\right\rangle_{\partial \Omega^{m}}^{2}}
$$

Use the same estimate as in Theorem 4 for all other terms, we get

$$
\begin{aligned}
\sum_{m} w_{m} A_{m}\left(e_{m}, \tilde{e}_{m}\right) \leq & \frac{D}{4}\left[\|e\|^{2}+\sum_{m} w_{m}\left(\left\langle\left\langle e_{m}^{+}-e_{m}^{-}\right\rangle\right\rangle_{\Gamma_{h}^{m}}^{2}+\frac{1-\sigma^{\prime}}{2}\left\langle\left\langle e_{m}\right\rangle\right\rangle_{\partial \Omega^{m}}^{2}\right)\right] \\
& +C h^{2 k+1}|\phi|_{k+1}^{2} .
\end{aligned}
$$


Combining (3.46) and (3.47) we have

$$
\|e\| \leq C h^{k+1 / 2}|\phi|_{k+1} .
$$

Again, $C$ always denote some generic constant in the proof.

Similarly as before, the main difficulty to extend the above proof for the reflection boundary condition in 3D is due to: (1) the difficulty for obtaining the similar inequality as (2.9) in some pathological case when $\hat{s}_{m}$ is almost tangential to the boundary at $\vec{x}$, and (2) the non-uniformity of the angular mesh on the sphere, i.e., the failure for establishing the similar claim in Lemma 4.

3.3. Spatial discretization by FDM. Here we only consider 2D FDM (2.4) with $\theta_{m} \in\left[0, \frac{\pi}{2}\right)$, which can be easily extended to other $2 \mathrm{D}$ cases or $3 \mathrm{D}$ cases. Here, let $\|f\|_{\infty}:=\max _{i, j, m}\left|f_{i, j, m}\right|$, the max norm with respect to both angular and spatial variables. Without loss of generality, assume $\triangle x=\Delta y=h$. Then we have the following result on the error estimate of FDM (2.4) for (1.1).

Theorem 6. Assume the solution to (1.1) $\Phi(\vec{x}, \hat{s})$ is $C^{2}(\Omega \times S)$ and denote $e_{i, j, m}:=$ $\phi_{i, j, m}^{h}-\Phi\left(x_{i}, y_{j}, \hat{s}_{m}\right)$, where $\phi_{i, j, m}^{h}$ is the numerical solution from (2.4), then

$$
\|e\|_{\infty}=O(h)+O\left(h_{a}^{2}\right) .
$$

Proof. For non-boundary nodes, $e_{i, j, m}$ satisfies

$$
\left(a+b+\mu_{t}\right) e_{i, j, m}-\left(a e_{i-1, j, m}+b e_{i, j-1, m}\right)-\mu_{s} \sum_{m^{\prime}} w_{m m^{\prime}} e_{i, j, m^{\prime}}=T_{i, j, m}
$$

where the local truncation error $T_{i, j, m}=O(h)+O\left(h_{a}^{2}\right)$ due to first order upwind difference in space and piecewise linear approximation in angle (Theorem 1).

Let $e_{i, j, m}=\|e\|_{\infty}$. Since $a+b+\mu_{t}, a, b$ and $\mu_{s} w_{m m^{\prime}}$ are all non-negative, and $\left(a+b+\mu_{t}\right)-\left(a+b+\mu_{s} \sum_{m^{\prime}} w_{m m^{\prime}}\right)=\mu_{a}>0$, at $(i, j, m)$, the linear system has an $M$-matrix and we have

$$
\left(a+b+\mu_{t}\right)\|e\|_{\infty} \leq a\|e\|_{\infty}+b\|e\|_{\infty}+\mu_{s} \sum_{m^{\prime}} w_{m m^{\prime}}\|e\|_{\infty}+\left|T_{i, j, m}\right| .
$$

Since $\sum_{m^{\prime}} w_{m m^{\prime}}=1$, we have

$$
\mu_{a}\|e\|_{\infty} \leq\|T\|_{\infty}
$$

Let $C$ be the positive lower bound of $\mu_{a}$, thus we have proved the statement that

$$
\|e\|_{\infty} \leq \frac{1}{C}\|T\|_{\infty}=O(h)+O\left(h_{a}^{2}\right) .
$$

For boundary nodes with general discretized reflection boundary condition (2.8),

- if $\hat{s}_{m} \cdot \hat{n}>0$, i.e., $\left(x_{i}, y_{j}, \hat{s}_{m}\right) \in \Gamma^{+}$, after we use relation (2.8) to substitute all $e_{i, j, m^{\prime}}$ with $\hat{s}_{m^{\prime}} \cdot \hat{n} \leq 0$, we have

$$
\begin{aligned}
& \left(a+b+\mu_{t}\right) e_{i, j, m}-\left(a e_{i-1, j, m}+b e_{i, j-1, m}\right)-\mu_{s}\left(\sum_{m^{\prime}: \hat{s}_{m^{\prime}} \cdot \hat{n}>0} w_{m m^{\prime}} e_{i, j, m^{\prime}}\right. \\
& \left.\quad+\sum_{m^{\prime}: \hat{s}_{m^{\prime}} \cdot \hat{n} \leq 0} w_{m m^{\prime}} \sum_{m^{\prime \prime}: \hat{s}_{m^{\prime \prime}} \cdot \hat{n}>0} r_{m^{\prime} m^{\prime \prime}} e_{i, j, m^{\prime \prime}}\right)=T_{i, j, m}
\end{aligned}
$$

Here $T_{i, j, m}$ may include the error due to linear interpolation of the exact reflection boundary condition, which is of order $O\left(h_{a}^{2}\right)$. Since $r_{m^{\prime} m^{\prime \prime}} \geq 0$ and $\sum_{m^{\prime \prime}} r_{m^{\prime} m^{\prime \prime}} \leq 1$, the $M$-matrix system is preserved by our angular discretization at the boundary. The same argument used above shows $\|e\|_{\infty}=O(h)+O\left(h_{a}^{2}\right)$. 
- If $\hat{s}_{m} \cdot \hat{n} \leq 0$, from boundary condition (2.8) we have

$$
e_{i, j, m}=\sum_{m^{\prime}: \hat{s}_{m^{\prime}} \cdot \hat{n}>0} r_{m m^{\prime}} e_{i, j, m^{\prime}}+T_{i, j, m} .
$$

Again, $T_{i, j, m}$ may include the error due to linear interpolation of the exact reflection boundary condition.

Combining the above cases, we see that $\|e\|_{\infty}=O(h)+O\left(h_{a}^{2}\right)$ is also true for boundary nodes.

Since the fully discretized linear system has a $M$-matrix structure, the ISI (2.6), which is a Gauss-Seidel iteration, converges and converges faster than the standard SI (2.7). Here is a simple argument.

Let $E^{n}:=\left\|\phi_{i, j, m}^{n}-\phi_{i, j, m}^{h}\right\|_{\infty}$, by the similar argument in Theorem 3, for ISI (2.6), we have

$$
E^{n} \leq \frac{r \sum_{m^{\prime}=m+1}^{M} w_{m m^{\prime}}}{1+r \sum_{m^{\prime}=m+1}^{M} w_{m m^{\prime}}} E^{n-1}=\left(1-\rho_{m}\right) E^{n-1},
$$

where $r=\left\|\mu_{s} / \mu_{a}\right\|_{\infty}$ and $\rho_{m}=\frac{1}{1+r \sum_{m^{\prime}=m+1}^{M} w_{m m^{\prime}}}$.

Similarly, for SI (2.7), we have

$$
E^{n} \leq \frac{r \sum_{m^{\prime}=1}^{M} w_{m m^{\prime}}}{1+r \sum_{m^{\prime}=1}^{M} w_{m m^{\prime}}} E^{n-1}=\left(1-\rho_{J}\right) E^{n-1}
$$

with $\rho_{J}=\frac{1}{1+r}$. Obviously, ISI converges faster than SI since $\rho_{m}>\rho_{J}$.

When scattering is weak, e.g., $r=\left\|\mu_{s} / \mu_{a}\right\|_{\infty} \ll 1$, or in the case of forwardpeaking regime, which is usually the case in optical imaging, e.g., $g \sim 1$ in H-G, $1-w_{m m} \ll 1$, it can be seen easily from the above argument that the improvement of ISI over SI is significant. In the extreme case with $g=1, w_{m m^{\prime}}=0$ for $m \neq m^{\prime}$, ISI takes only one iteration when the spatial ordering is along the characteristic for each transport direction. When scattering is dominant $(r>>1)$ and $f$ is isotropic, or the domain is optical thick, even ISI may converge quite slow. Since ISI captures transport effectively and is a smoothing operation, it is used as a natural relaxation scheme for a multigrid algorithm in both space and angle [4, which can deal with RTE in different regimes effectively.

\section{REFERENCES}

1. Cockburn B., Dong B., and Guzmán J., Optimal convergence of the original discontinuous Galerkin method for the transport-reaction equation on special meshes, SIAM J. Numer. Anal. 3 (2008), 1250-1265. MR2390992(2009c:65299)

2. Johnson C. and Pitkäranta J., An analysis of the discontinuous galerkin method for a scalar hyperbolic equation, Math. Comp. 46 (1986), 1-26. MR.815828 (88b:65109)

3. Richter G. R., An optimal-order error estimate for the discontinuous galerkin method, Math. Comp. 50 (1988), 75-88. MR917819 (88j:65197)

4. Gao H. and Zhao H. K., A fast forward solver of radiative transfer equation, Transport Theory and Statistical Physics 38 (2009), 149-192. MR2572432 (2011b:85008)

5. Jackson J. D., Classical electrodynamics, 3rd edition, Wiley, New York (1999).

6. Case K. M. and Zweifel P. F., Linear transport theory, Addison-Wesley, Massachusetts (1967). MR0225547 (37:1140)

7. Lesaint P. and Raviart P. A., On a finite element method for solving the neutron transport equation, Mathematical aspects for finite elements in partial differential equations, Academic Press, New York (1974). MR0658142 (58:31918) 
8. Han W., Huang J., and Eichholz J. A., Discrete-ordinate discontinuous galerkin methods for solving the radiative transfer equation, SIAM Journal on Scientific Computing 32 (2010), 477497. MR.2609327 (2011f:65198)

9. Reed W. H. and Hill T. R., Triangular mesh methods for the neutron transport equation, Los Alamos Scientific Laboratory Report LA-UR-73-479 (1973).

Department of Mathematics, University of California, Los Angeles, California 90095-1555

E-mail address: haog@math.ucla.edu

Department of Mathematics, University of California, Irvine, California 92697-3875

E-mail address: zhao@math.uci.edu 